\title{
AN R.F. INPUT COUPLER SYSTEM FOR THE CEBAF ENERGY UPGRADE CRYOMODULE *
}

\author{
J. R. Delayen, L.R. Doolittle, T. Hiatt, J. Hogan, J. Mammosser, L. Phillips", J. Preble, \\ W.J. Schneider, G. Wu, Jefferson Lab, Newport News, VA
}

\section{Abstract}

Long term plans for CEBAF at Jefferson Lab call for achieving $12 \mathrm{GeV}$ in the middle of the next decade and 24 $\mathrm{GeV}$ after 2010. Thus an upgraded cryomodule to more than double the present voltage is under development. A new waveguide coupler system has been designed and prototypes are currently being developed. This coupler, unlike the original, has a nominal $Q_{\text {ext }}$ of $2.1 \times 10^{7}$, reduced sensitivity of $Q_{\text {ext }}$ to mechanical deformation, reduced field asymmetry within the beam envelope, freedom from window arcing with a single window at 300 $\mathrm{K}$, and is capable of transmitting $6 \mathrm{~kW} \mathrm{CW}$ both traveling wave and in full reflection.

\section{INTRODUCTION}

The CEBAF upgrade baseline design calls for $1500 \mathrm{MHz}$, 7-cell cavities operating at $12.1 \mathrm{MV} / \mathrm{m}$ with a circulating current up to $400 \mu \mathrm{A}$. [1] To minimize cost, our goal is only a modest increase of the RF power available to each cavity from 5.5 to $6 \mathrm{~kW}$. This puts stringent constraints on the maximum amount of detuning (static and dynamic) and on coupling system $Q_{\text {ext accuracy. }}$ Figure 1 shows the of RF power required as a function of $Q_{\text {ext }}$ at the gradient of $12.5 \mathrm{MV} / \mathrm{m}$, detuning of $25 \mathrm{~Hz}$ and for circulating currents of 0,200 , and $400 \mu \mathrm{A}$. The optimal $Q_{\text {ext }}$ is $2.1 \mathrm{x}$ $10^{7}$ with an acceptable range of 1.5 to $3 \times 10^{7}$

Both coaxial and waveguide couplers were explored. The waveguide concept was retained for its simplicity and flexibility at $1500 \mathrm{MHz}$. Unlike the present design, however, the fundamental power coupling (FPC) and

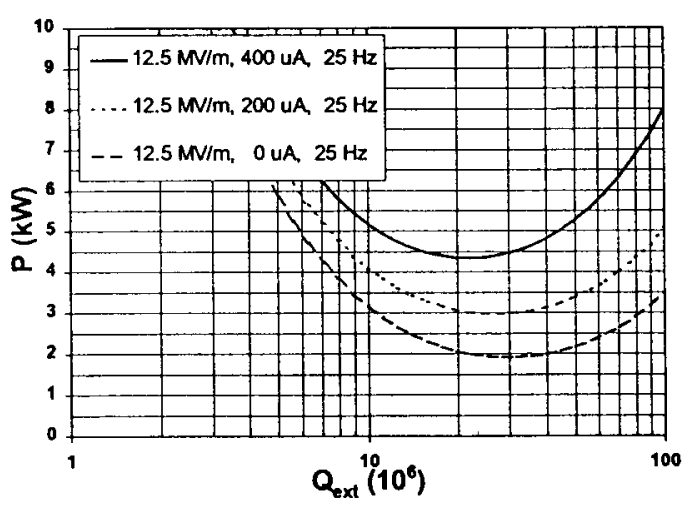

Figure 1

\footnotetext{
* Supported by U.S. DOE contract DE-AC05-84-ER40150

"Email: phillips@jLab.org
}

higher order mode (HOM) extraction functions are completely separate. This produces a coupler design that, unlike the present one, is free of transverse beam kick, and allows a cryostat design with all the power couplers on the same side.

\section{THE CAVITY-COUPLER SYSTEM}

The nominal cavity gradient is $12.5 \mathrm{MV} / \mathrm{m}$ with a beam current of $400 \mu \mathrm{A}$. A shorted waveguide intercepting the beam pipe couples $R F$ power to the evanescent $T_{M}$ mode of a 7-cell cavity (Figure 2). A copper-plated stainless steel waveguide provides a thermal transition between the cavity at $2 \mathrm{~K}$ and the outer cryostat envelope at $300 \mathrm{~K}$. The cavity vacuum extends to a single rectangular waveguide window at $300 \mathrm{~K}$.

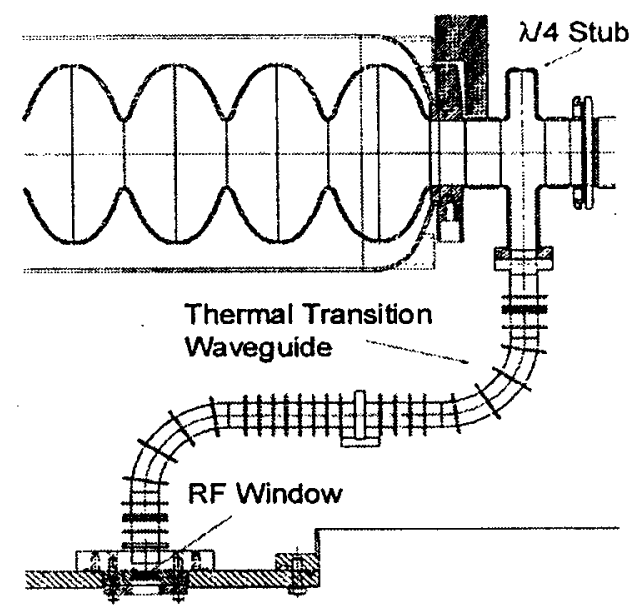

Figure 2

The new beam line configuration reflects several changes. With no gatevalves or bellows between cavities, cavity length increased from five to seven cells. This requires both lateral and radial flexibility in the waveguide thermal transition to allow for thermal differential contraction between the cavity string and the outer vacuum envelope, the accumulated cavity motion arising from cavity tuner operation, and manufacturing tolerances. The longitudinal motion of the string of eight cavities will be fixed at the center of the cryomodule requiring a displacement at each end amounting to 1.3 $\mathrm{cm}$. This motion is provided by three short waveguide bellows appropriately placed. 
The cavities' flexible waveguides and warm windows are attached in the clean room, with the entire cavity string then permanently evacuated for all subsequent cryomodule assembly. As shown, each waveguide has two $90^{\circ}$ elbows. This accomplishes two purposes: reduction of radial penetration ports from eight to four, and avoidance of window charging leading to "arc" trips by removing the ceramic window from the cavity fields' radiation flux. Currently $40 \%$ of CEBAF cavities are limited in gradient by field-emission-induced "arcing." Tests have shown that moving the RF window from a direct line-of-sight view of the high field region of the cavity can eliminate field-emission-induced window "arcs." The need to offset the window to prevent "arcing" and the need for longitudinal motion were factors favoring the simplicity of a waveguide coupler over a coaxial design.

\section{BEAM LINE COUPLER}

The cavity-coupling factor was chosen to be $2.1 \times 10^{7}$ (Figure 1). A $\lambda / 4$ stub waveguide coupler intersecting the beam pipe couples the $\mathrm{TE}_{01}$ waveguide mode to the evanescent $\mathrm{TM}_{01}$ cavity mode. The waveguide-couplerto-cavity separation was selected to achieve the desired coupling. The Cornell/CEBAF cavity currently used has a $\lambda / 2$ stub waveguide coupler and was originally designed for storage ring applications. The $\lambda / 2$ stub coupler provided a $Q_{\text {ext }}$ of $3 \times 10^{5}$ and was located up against the iris of an end cell. Coupling to the fundamental can occur with the stub length slightly less than $\lambda / 2$, while $\mathrm{TE}_{111}$ cavity modes in the 1.7 to $1.9 \mathrm{GHz}$ range, which are beyond cut-off of the waveguide HOM couplers, are even more strongly damped. This feature is not relevant for the energy upgrade cavity, which will have coaxial HOM dampers. Furthermore, there are several disadvantages to the $\lambda / 2$ coupler over the $\lambda / 4$ coupler. Locating the beam line close to the null in the standing wave pattern makes the coupler $Q_{\text {ext }}$ quite sensitive to small shifts in this pattern due to manufacturing tolerances and subsequent waveguide deformations. This becomes even more acute for the higher fundamental rejection required for $Q_{\text {ext }}$ of $2.1 \times 10^{7}$ as opposed to $3 \times 10^{5}$. Another drawback is the field asymmetry across the beam pipe giving rise to a "coupler kick"

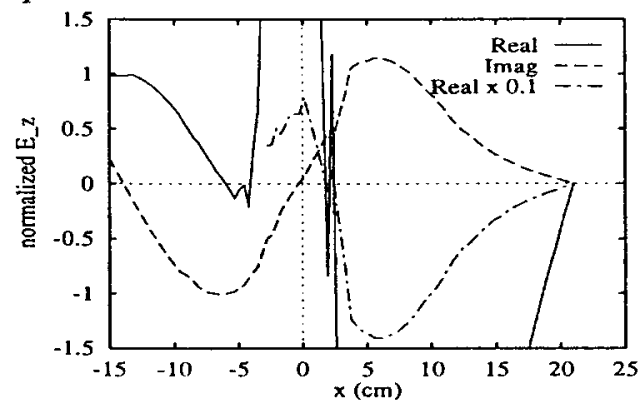

Figure 3: Electric fields in the centerline of $\lambda / 2$ stub-onstub PC used by the Cornell/JLab cavity system [2]
In CEBAF this effect was reduced by alternating the longitudinal coupler orientation in a pattern averaging the net kick to an acceptable level. [2], [3]

We have chosen the $\lambda / 4$ stub waveguide coupler for the 7 -cell energy upgrade cavity. (Figure 4 show the electric field along the FPC waveguide centered on the beam line for this configuration. [2] In addition to eliminating the "coupler kick," the reduced coupler fields will contribute negligible heat to the coupler body outside the helium envelope

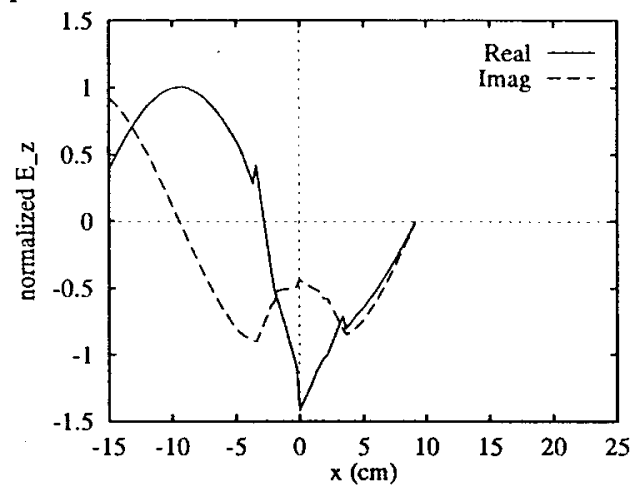

Figure 4: Electric fields in the centerline of $\lambda / 4$ stub FPC proposed for the CEBAF energy upgrade cryomodule. [2]

The sensitivity of $Q_{\text {ext }}$ of the FPC to mechanical deformation and variations in manufacturing tolerances has also been reduced significantly. We have used this insensitivity by allowing the FPC waveguide to take up cavity misalignment and axial beam line displacement without significant change in the cavity coupling factor. The $Q_{\text {ext }}$ variation with axial deformation (i.e., reducing or increasing the height of the FPC waveguide at the beam pipe) is $6 \% / \mathrm{mm}$ for small displacements. For angular displacements of the beam pipe away from the beamline, rotating about the center of the FPC waveguide, the $Q_{\text {ext }}$ variation is $0.15 \% / \mathrm{mrad}$. Consequently, the beamline between cavities is entirely niobium with no bellows. Some flexibility has also been included in the niobium flanges joining cavities. These two flexible elements are used to absorb all misalignment in the beamline.

\section{WAVEGUIDE THERMAL TRANSITION}

A copper-plated stainless steel waveguide provides the required thermal isolation between an RF window at 300 $\mathrm{K}$ and the cavity waveguide coupler flange at $2 \mathrm{~K}$, with a thermal intercept at $50 \mathrm{~K}$. The FPC refrigeration load at 2 $\mathrm{K}$ is the sum of thermal conduction and RF dissipation from the waveguide between $2 \mathrm{~K}$ and $50 \mathrm{~K}$, and is minimized, for a given waveguide structure, with respect to waveguide length.

The RF fields averaged circumferentially over a slice of waveguide perpendicular to the direction of propagation is shown in Figure 5 for normal operating conditions and in Figure 6 for the worst case condition.[4] 


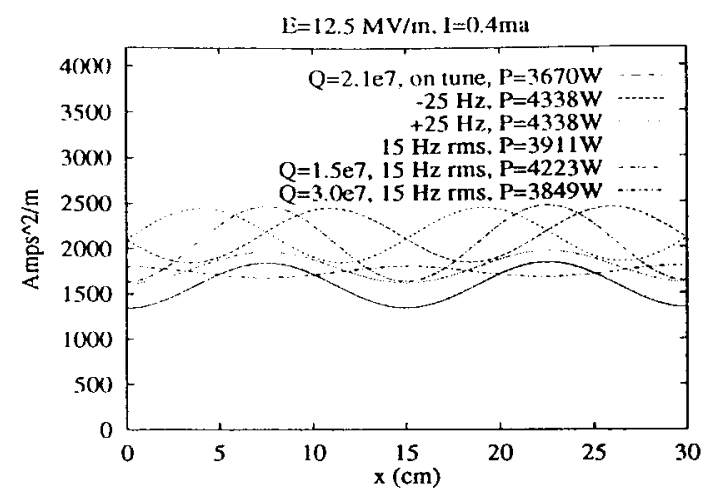

Figure 5: Typical currents on waveguide walls [4]

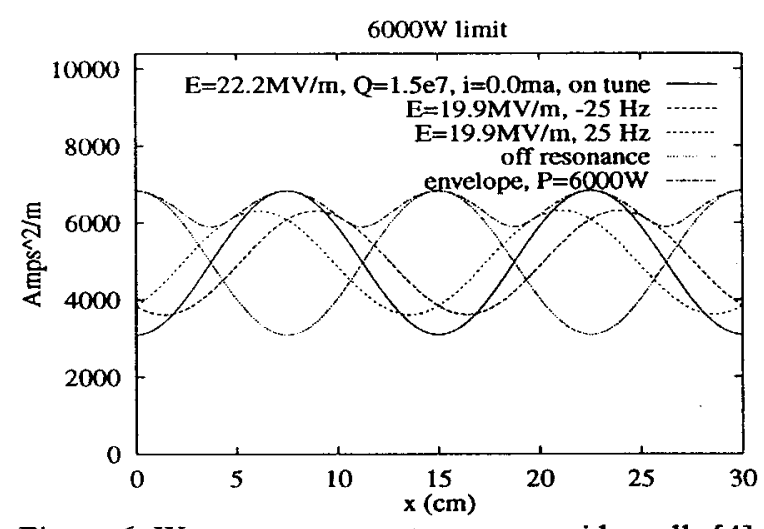

Figure 6: Worse case current on waveguide walls [4]

In balancing RF dissipation against heat conduction down the guide, a broad minimum in $2 \mathrm{~K}$ heat load as a function of waveguide length is seen (Figure 7).

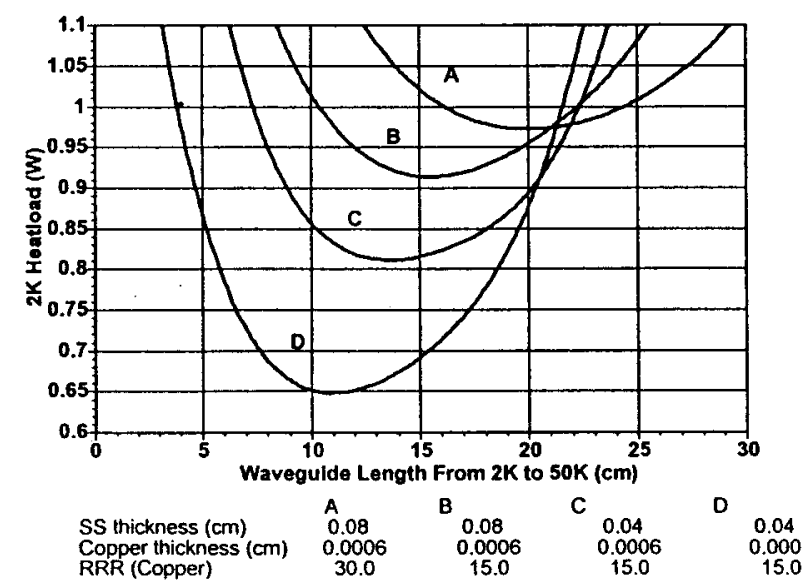

Figure 7

When operated at a minimum, the highest temperature along the waveguide will be at the high temperature end of the guide. Under worst case conditions the same waveguide will reach a peak temperature somewhere along the guide significantly higher than the end of the guide. The potential for sudden gas migration in such an excursion possibly inducing waveguide discharge will be studied with prototype components in a horizontal test cryostat later this year. Details of the copper plating will also be studied.

The heat load at $2 \mathrm{~K}$ will be less than $1 \mathrm{~W}$ under normal operating conditions. The minimum lengthoptimized heat load will depend on the total thermal conductance of the stainless steel waveguide walls and of the copper plating in addition to the RF loss in the copper. Although the surface impedance of the copper is predominantly in the anomalous limit between $2 \mathrm{~K}$ and 50 $\mathrm{K}$, the residual resistivity ratio (RRR) and thickness of the copper layer have a significant influence, and must be chosen to balance performance risk against overly conservative manufacturing tolerances. [5]

\section{RF WINDOW}

Each coupler contains one RF window at $300 \mathrm{~K}$. The window is a thin metal-ceramic waveguide window using a rectangular copper-gasketed knife-edge seal on the cavity vacuum side. A variety of similar windows have been developed at JLab having RF power handling capabilities in excess of the CEBAF energy upgrade requirements. The VSWR is less than 1.1 to 1 through the use of compensating irises built into the window.

\section{SUMMARY}

A fundamental power coupler prototype has been designed for the CEBAF Energy Upgrade Cryomodule having several advantages over the existing design; most notably, the elimination of window arcs and unwanted beam steering from "coupler kicks". Prototype fabrication has begun and testing is scheduled later this year.

\section{ACKNOWLEDGES}

The authors wish to thank this staff of the Accelerator Development group and in particular the support of Sherry Thomas, Carolyn Camp, and Kelly Hanifan in the preparation of this work.

\section{REFERENCES}

[1] J. R. Delayen, "Upgrade of the CEBAF Acceleration System", these Proceedings.

[2] L. R. Doolittle, "Strategies for Waveguide Coupling for SRF Cavities", Proceedings of Linac98, Chicago, IL.

[3] R. C. York, C. Reece, "RF Steering in the CEBAF CW Superconducting Linac", Proceedings of the 1987 Particle Accelerator Conference, Washington, D.C

[4] L. R. Doolittle, "Waveguide Surface Currents", Jefferson Lab Technote.

[5] L. Phillips, G. Wu, JLab Technote to be published, "Material Properties and Thermal Transitions in RF Power Couplers". 